\title{
Trauma and General Surgeon
}

\section{Satish Dharap ${ }^{1}$}

Published online: 16 March 2020

(C) Association of Surgeons of India 2020

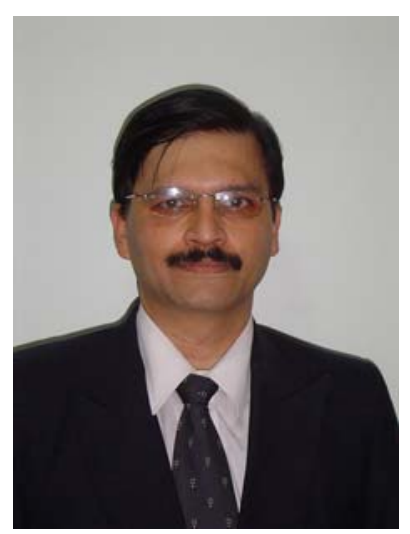

Trauma, whether accidental or intentional injury, is today a major healthcare issue. In developed countries, trauma is the leading cause of death in the first four decades of life and is a top contributor to the disability-adjusted life years (DALYs) lost. The problem has assumed an epidemic proportion in India. According to the Ministry of Road Transport and Highways, 147,913 people were killed and 470, 975 persons were injured in 464,910 road crashes in India in 2017. This translates to 1273 crashes and 405 deaths every day or 53 crashes and nearly 17 deaths every hour. The number of road crash deaths has increased by $23.4 \%$ from 2008 to 2017 [1]. With wide highways, faster vehicles and poor road discipline, road traffic crashes have been responsible for $45.1 \%$ accidental deaths in 2017 [2]. The problem is not only urban but encompasses all regions of the country. To control mortality and morbidity of injury, many developed countries have evolved trauma systems. A trauma system is a holistic approach that encompasses the prevention, access to care, prehospital care, hospital care which includes trauma centers

Satish Dharap

drdharap@Hotmail.com

1 TN Medical College, BYL Nair Hospital, Mumbai 400 008, India of different categories, rehabilitation, education, and disaster care.

Many states today do have a public ambulance system providing some prehospital care. Are we ready to treat critically injured patients? The honorable Supreme Court has made it mandatory for all hospitals to give basic care to all accident victims before transfer. At many medical colleges, exclusive trauma centers are being developed; however, the number is not enough considering the magnitude of the problem. Well-equipped stand-alone trauma centers having specialist trauma surgeons often remain without much work and appear as a waste of resources. This can be avoided by developing trauma and emergency medicine departments or services in tertiary care hospitals, with the sharing of expensive resources. Some medical colleges and some private tertiary care hospitals have well-established emergency departments with emergency physicians trained in triage and initial resuscitation. Many other hospitals including some medical colleges still have a casualty department manned by casualty officers who do not have any specialized training in managing emergencies and refer patients to the specialty departments for further management. However, an injured is usually taken to the nearest hospital. Perhaps, the largest number is treated in local primary or secondary health centers or private hospitals and nursing homes, most of which lack basic minimum infrastructure and trained manpower to handle emergencies. These deficiencies may be contributing to trauma deaths which are potentially preventable, and some centers have reported a high rate of unexpected deaths [3-5].

To improve patient care and outcomes in general, modern medical education envisages specialization in different fields. We have witnessed specializations in several fields over the last five decades. There are specializations of body systems (such as urology, neurosurgery, cardiac surgery, vascular surgery, hepato-biliary and pancreatic surgery, gastrointestinal surgery, endocrine surgery, head and neck surgery), body reconstruction (plastic and esthetic surgery), and age (pediatric surgery). There is also specialization depending upon diseases (surgical oncology) and type of access and technique (minimal access and endoscopic surgery and micro-vascular surgery). 
There can be further specialization cutting across these specializations, e.g., robotic uro-onco-surgeon or a multi-organ transplant surgeon. Each specialization involves a focused approach and skill development in a specific field. The purpose of specialization is not only to enhance skills but also to promote research, education, and professional development. Specialization makes it feasible to perform a constant update of knowledge and skills, which is necessary to maintain professional excellence.

So, should we be having specialized trauma surgeons? Such a policy has also been considered for trauma care, and super-specialty degree or fellowship courses in trauma surgery are available in some premier tertiary care institutions. However, unlike other specializations, trauma surgery involves further broadening of scope. It is said that "to train a specialist in the treatment of trauma is to train a specialist in all of the specialties" [6]. Also, specialist trauma surgeons are available only in trauma centers in some tertiary care teaching hospitals. The present capability of specialized training in trauma surgery is very limited and is not enough to address the present patient load.

So how can trauma care be improved? It is important to understand the pathophysiology and principles of management of trauma patients which have evolved therefrom. Trauma leads to anatomical disruption of one or more body systems and at times leads to acute physiological derangements immediately threatening the patient's life. In such patients, survival depends on timely treatment, i.e., efficiency of the doctor on the spot in recognizing and treating immediately life-threatening injuries. The key is the capacity building, and one such global effort is the Advanced Trauma Life Support (ATLS) program of the American College of Surgeons, which is available in many countries including India. Algorithmbased drills as professed in ATLS have shown a reduction in deaths and morbidity from trauma. Some centers in India and abroad have alternative training programs based on similar principles. However, such training is not mandatory in India for doctors working in emergency departments. The majority of life-threatening injuries involves injuries to head, abdomen, chest, pelvis, and crush injuries involving lower extremities. So, to improve trauma care, efficient resuscitation, expeditious life-saving surgery, and care of the critical patient are needed. It involves teamwork and coordination among different specialists. Today, specialist trauma surgeons are available in very few hospitals. In many hospitals, triage and initial resuscitation are done by emergency physicians and a patient with multiple injuries is transferred under the care of an intensivist for further management. An intensivist is trained in managing a critical patient; however, it is not trained to perform immediate life-saving operations. Therefore, to manage a trauma patient with multiple injuries, a team leader who is a versatile medical professional with excellent basic and broad-based surgical training is needed. Most opinions consider that a general surgeon is most suited to lead a trauma team [6-8].

Literature from developed countries as well as India has revealed better outcomes in patients directly going to specialized trauma units or centers [9-11]. However, trauma patients do not have a choice of the hospital as there are very few such centers in India and those are also located mainly in large cities. The reason why such centers have a better outcome is their organized approach towards the management of trauma patients consisting of triage, initial assessment and resuscitation, life-saving surgery, determination of priorities of definitive care, coordination among different specialists for definitive care of systemic injuries, critical care of such patients, and rehabilitation.

As most trauma patients are initially taken to smaller local hospitals, training of doctors on duty in the emergency department and development of trauma team leaders capable of performing life-saving surgeries and coordinating with different specialists are needed and an exclusive specialist trauma surgeon is not necessary. A general surgeon is most suitable to step into the shoes of a trauma surgeon and trauma team leader. He needs some grooming to perform initial resuscitation of a patient with multiple injuries to conduct life-saving interventions and damage control surgery in an unstable patient. Often, an obvious injury distracts the attention and an internal injury that could be life-threatening is missed. By training, such errors can be easily avoided. Missed injuries have been observed to be significantly lower when a surgeon is the team leader [12]. Understanding of dos and don'ts of non-operative management and the role of interventional radiology and timely surgery needs some experience. A team leader also needs the training to determine the priorities of definitive treatments in a patient with multiple injuries. At times, in a trauma patient, it is difficult to determine the dominant injury and therefore the specialty responsible to care for the patient. General surgery should be a default specialty to take care of any such patient, which can avoid unnecessary arguments in the emergency department. The trauma team leader also needs to know about basic critical care. Critically injured patients need prolonged care. An unconscious patient may need tracheostomy for the tracheo-bronchial toilet, a procedure for feeding, preventive care for pressure sores, physiotherapy, and rehabilitation. A general surgeon has an important role in taking the appropriate decision and maintaining continuity of care.

All the resources required for the care of the injured may not be available locally. Considering the patient's needs and the available resources, the trauma team leader has to take the decision to transfer to the appropriate center. This involves prior communication with the higher center and provision of care during transport to ensure a safe transfer. In the USA, hospitals caring for the injured are categorized into five levels 
depending on the availability of resources - level V being the center with only the basic resuscitative facilities (similar to a primary health center in India) to level I being a $24 \times 7$ multispecialty hospital with all modern amenities including teaching (similar to tertiary care medical college with post-graduate program). There are also well-established transfer protocols for inter-hospital transport. Centers designated by local governments are verified by the American College of Surgeons for quality [13].

Making a trauma surgeon thus involves the training of a general surgeon in trauma management skills; which include initial resuscitation, life-saving and definitive surgery, nonoperative management, critical care, and coordination and communication. Structured courses are available for training in initial management, definitive care, and critical care. These courses emphasize systematically approaching a patient and involve unlearning and relearning and reorganization of the thought process. The most important attributes which make a good trauma team leader are the qualities collaboration, communication, and decisiveness [14].

Thus, the development of trauma team leadership by educating general surgeons has the potential of improving trauma care in India. Undoubtedly, the management of trauma should be an integral part of the training of every general surgeon. The principles of management of acute trauma are valid not only while managing the injured patients but in all other surgical fields as well, and this training goes a long way in boosting overall surgical skills and capability.

\section{References}

1. https://savelifefoundation.org/wp-content/uploads/2018/10/RoadCrash-Statistics-2017.pdf Retrieved on 17 February 2020
2. http://ncrb.gov.in/StatPublications/ADSI/ADSI2017/chapter-A\% 20traffic\%20accidents.pdf Retrieved on 17 February 2020

3. Uthkarsh PS, Gururaj G, Sai Reddy S, Rajanna MS (2016) Assessment and availability of trauma care services in a district hospital of South India; a field observational study. Bull Emerg Trauma 4(2):93-100

4. Kumar S, Chaudhary S, Kumar A, Agarwal AK, Misra MC (2009) Trauma care-a participant observer study of trauma centers at Delhi. Lucknow and Mumbai Indian J Surg 71(3):133-141

5. Goel A, Kumar S, Bagga MK (2004) Epidemiological and trauma injury and severity score (TRISS) analysis of trauma patients at a tertiary care centre in India. Natl Med J India 17(4):186-189

6. Allgöwer M (1975) General surgery and trauma. Ann R Coll Surg Engl 57(3):133-138

7. Pierce GE (1991) Trauma surgery: every surgeon's specialtypresidential address. Western Trauma Association J Trauma 31(12):1575-1578

8. Reddy BR (2011) Debate on who should lead the trauma teamsurgeon vs. intensivist? J Med Allied Sci 1(2):90-92

9. Dharap SB, Kamath S, Kumar V (2017) Does prehospital time affect survival of major trauma patients where there is no prehospital care? J Postgrad Med 63(3):169-175

10. Sampalis JS, Denis R, Fréchette P, Brown R, Fleiszer D, Mulder D (1997) Direct transport to tertiary trauma centers versus transfer from lower level facilities: impact on mortality and morbidity among patients with major trauma. J Trauma 43:288-295

11. Cheddie S, Muckart DJ, Hardcastle TC, Den Hollander D, Cassimjee H, Moodley S (2011) Direct admission versus interhospital transfer to a level I trauma unit improves survival: an audit of the new Inkosi Albert Luthuli Central Hospital trauma unit. S Afr Med J 101:176-178

12. Leeper WR, Leeper TJ, Vogt KN, Charyk-Stewart T, Gray DK, Parry NG (2013) The role of trauma team leaders in missed injuries: does specialty matter? J Trauma Acute Care Surg 75(3):387-390

13. https://www.amtrauma.org/page/traumalevels Retrieved on 17 February 2020

14. Mo D, O'Hara NN, Hengel R, Cheong AR, Singhal A (2019) The preferred attributes of a trauma team leader: evidence from a discrete choice experiment. J Surg Educ 76(1):120-126

Publisher's Note Springer Nature remains neutral with regard to jurisdictional claims in published maps and institutional affiliations. 